\title{
Generalizing the Biomimetic Design Process
}

\author{
L.H. Shu, Associate Professor \\ Dept. of Mechanical \& Industrial Engineering, University of Toronto \\ shu@mie.utoronto.ca
}

\begin{abstract}
While many elegant solutions to engineering problems have been inspired by biological phenomena, it is not always clear how the particular biological phenomena were selected. The objective of this work is to develop a generalized methodology by which analogous biological phenomena can be identified and used for any design problem in a systematic manner. The chosen approach has been implemented in a computer search tool that locates in a biology text, instances of functional keywords describing the engineering problem. Analogies for case studies in design for remanufacture and microassembly as well as ongoing work will be summarized.
\end{abstract}

\section{Introduction}

There are many elegant solutions to engineering problems that were inspired by biological phenomena. Most work in biomimetic design involves specific cases of design that copy particular biological models. For example, topics submitted to this symposium include robots modeled after insects and other organisms, and leaves serving as models for antennas and vascular networks. Biological models are also used to develop novel materials, actuators and sensors, some for prosthetic or implant applications. However, not always described is how particular biological models were identified or selected. Therefore, an engineer open to using biological models for design may not know how to find relevant biological analogies for a given design.

This paper will summarize our past and current work towards developing an approach to identify and use relevant biological analogies for any given engineering problem. Analogies identified using this approach for case studies in design for remanufacture and microassembly will be described. Finally discussed will be ongoing challenges and work towards overcoming these challenges.

\section{Methods}

The goal of this work is to develop a generalized methodology by which analogous biological phenomena can be identified and used for any engineering design problem in an objective and repeatable manner. One possible approach to enable generalized biomimetic design is to build a database of biological phenomena for engineering use (Vincent and Mann, 2002). The approach described here is fundamentally different and avoids the enormous and possibly subjective task of cataloguing biological phenomena for engineering. Instead, this method takes advantage of the abundant biological information already available in natural language format by searching it directly for relevant phenomena.

This approach has been implemented in the form of a computerized search tool that locates in biology texts, instances of functional keywords describing the engineering design problem.

\section{a. Source}

The initial source of information selected is Life, the Science of Biology (Purves et al., 2001), which is the reference text for the introductory course in biology at the University of Toronto. This text is suitable because it is written at a level that can be easily understood by those with little background in biology. Also, the text covers a large range of organizational levels, from the molecular and cellular, e.g., DNA, to the ecosystem, such that potential solutions are not limited to a particular organizational level.

\section{b. Keywords}

Keywords used to search for relevant text segments are verbs that describe the desired effect of possible solutions. Verbs are strongly preferred over nouns as keywords to initiate searches. Searching for nouns typically indicates pre-conceived solutions while searching for verbs that describe the desired action will identify biological forms that may not have occurred to the designer. 
Synonyms have been used in the past to increase the number of matches. More recent work has found that 'troponyms' produce superior alternative keywords. Troponyms are verbs that describe specific manners of another verb. For example, 'ambling' is a troponym of 'walking' because it is a particular manner of walking (Chiu and Shu, 2004).

\section{Examples}

Several analogies for case studies in design for remanufacture (Vakili and Shu, 2001, Hacco and Shu, 2002) and microassembly (Shu et al., 2003) were located at various levels of biological organization by searching for keywords and synonyms related to the engineering problem. These case studies will be briefly summarized.

\section{a. Design for remanufacture}

Remanufacture is a process applied to products at their end of life that seeks to reuse product components. Advantages over recycling for scrap material include conservation of resources required to melt and reform components. One design guideline identified to facilitate remanufacture is that product features that are prone to failure should be made separable (Shu and Flowers, 1999). In this way, the failed features can be replaced, enabling the reuse of a component without labor- and capital-intensive repair operations. However, making failure-prone parts separate increases part count and assembly cost. Biological analogies were sought to address this apparent contradiction between ease of assembly and ease of remanufacture.

Searching for the keyword 'remanufacture', not surprisingly, resulted in no matches in the biology text. In this case, alternative keywords such as synonyms are required to find any matches. Other keywords used include 'repair' and 'correct.'

Matches identified using 'repair' involve DNA repair mechanisms, including DNA proofreading during replication, mismatch repair and excision repair. Excision repair was found to be the most analogous to repairs performed during remanufacture. Text on excision repair follows (Purves et al. 2001):

An excision repair mechanism removes abnormal bases that have formed because of chemical damage and replaces them with functional bases.

... in excision repair, certain enzymes "inspect" the cell's DNA. When they find mispaired bases, chemically modified bases, or points at which one strand has more bases than the other (with the result that one or more bases of one strand form an unpaired loop), these enzymes cut the defective strand. Another enzyme cuts away the bases adjacent to and including the offending base, and DNA polymerase and DNA ligase synthesize and seal up a new (usually correct) piece to replace the excised one.

Although the occurrences of 'repair' in the matches are adjectives and nouns, they are still relevant to the verb 'repair' sought. Also, while the above excerpt confirms relevance of the excision repair phenomenon to remanufacture, the text does not provide ideas to solve our problem. Further research using a more advanced text, Friedberg et al. (1995), revealed details that could be used as stimuli for design.

\section{i. Extracting strategy from biological system}

Another phenomenon identified using the keyword 'correct' involves fainting. The text regarding fainting from Purves et al. (2001) follows:

\begin{abstract}
Blood must be returned from the veins to the heart so that circulation can continue. If the veins are above the level of the heart, gravity helps blood flow, but below the level of the heart, blood must be moved against the pull of gravity. If too much blood remains in the veins, then too little blood returns to the heart, and thus too little blood is pumped to the brain; a person may faint as a result. Fainting is self-correcting: A fainting person falls, thereby moving out of the position in which gravity caused blood to accumulate in the lower body.
\end{abstract}

The strategy derived from the above excerpt is that fainting is a form of defensive failure that prevents more serious failure.

\section{ii. Applying strategy to engineering system}

Snap fits embody a form of fastening often preferred for assembly and recycling purposes, but are problematic for remanufacture when they fail. Figures 1 and 2 show failed parts that contain snap fits in toner-cartridges undergoing remanufacture.

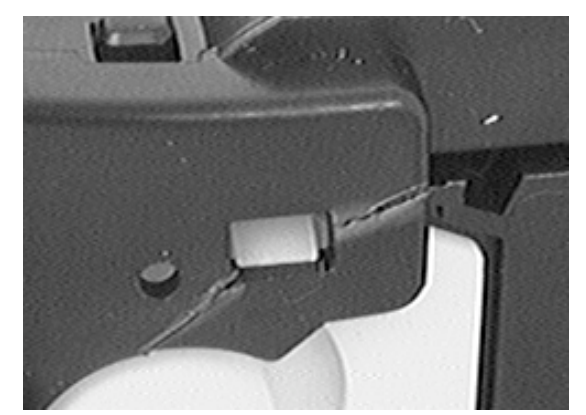

Figure 1. Damaged part with snap-fit, (Shu and Flowers, 1999). 


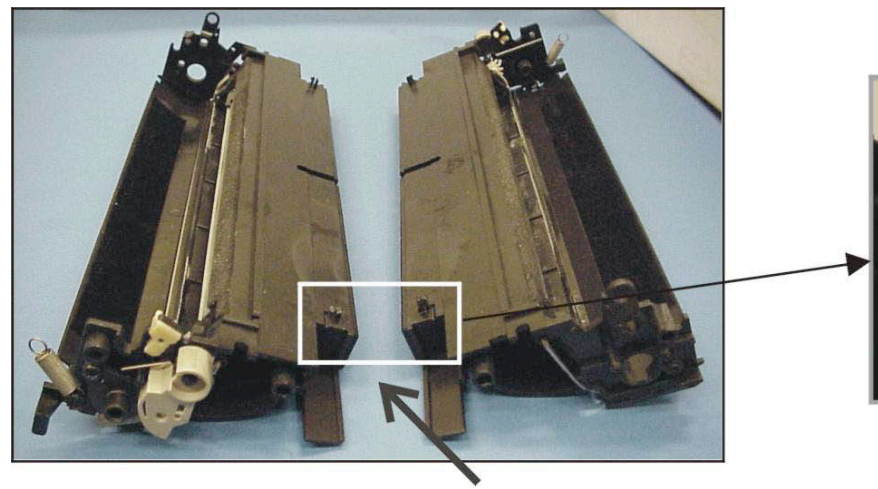

Enlarged Area

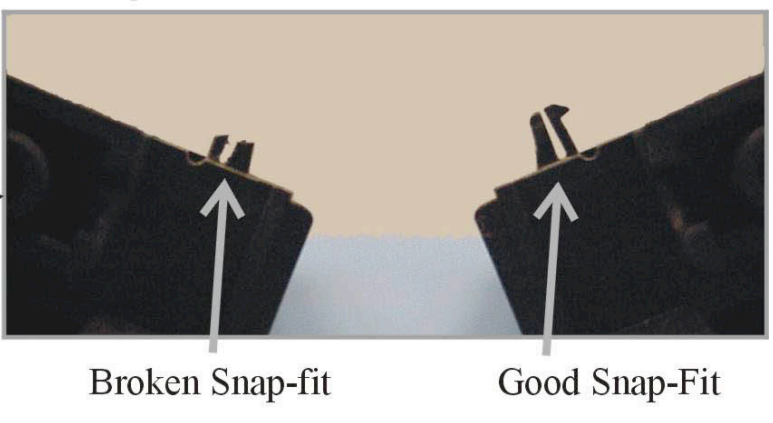

Viewpoint

Figure 2. Damaged snap-fit features (Williams, 2001).

Applying the fainting strategy to the design of snap fits, predetermined breakpoints incorporated into snap fit configurations as shown in Figure 3 may cause earlier failure than with standard snap fit configurations, but the part containing the snap fit feature can be more easily reused.

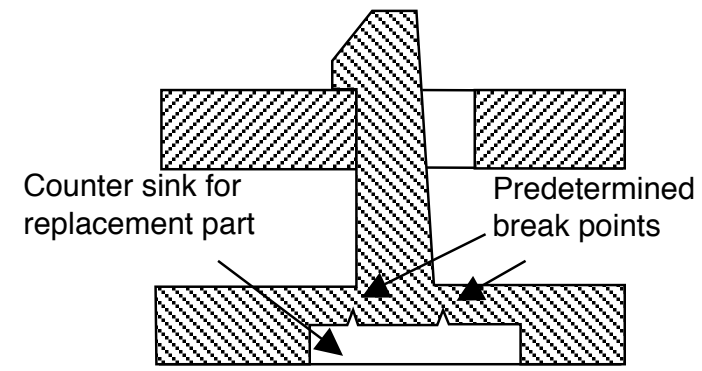

\section{a. Snap fit redesigned with counter sink and break points}

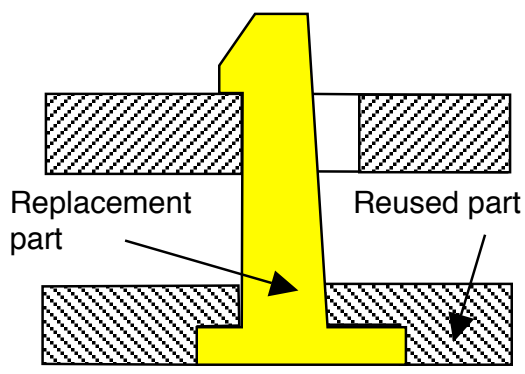

b. Redesigned snap fit after failure and refurbishment

Figure 3. Redesigned snap fit to facilitate repair (Hacco and Shu, 2002).

\section{b. Microassembly}

Collaboration with researchers with expertise in micro- and nanoengineering at the Technical University of Denmark led to the following case study in microassembly (Shu et al., 2003).

As the size of products and components become smaller, the manufacture and assembly of such components present challenges beyond those associated with their larger counterparts. For example, in the assembly of microobjects, additional challenges include the need for increased positional accuracy, the mechanics of interaction between objects, and the loss of direct hand-eye coordination.

Grippers used in microassemby can be roughly categorized as contact and non-contact. Contact grippers make use of principles including mechanical contact, vacuum, adhesion, electrostatics and ultrasound. Non-contact grippers make use of principles including optical traps, magnetic and electrical fields. Many of the aforementioned gripping techniques are reported to lack the ability to center objects. Miniaturized mechanical grippers can center objects but present problems including design complexity and not being suitable for clean rooms (Bütefisch and Büttgenbach, 2001).

The goal of the biomimetic search in this example is to identify biological phenomena relevant to the centering of objects within a gripper during microassembly. The functional keyword 'center' was used to locate relevant phenomena in Purves et al. (2001), including microtubule organizing centers, photosystems, and retinal ganglion cells (Shu et al., 2003). The current paper will only describe how the microtubule-organizing center (MTOC) centers itself in certain types of cells. First, the structure and function of microtubules will be outlined. 
Microtubules have the shape of hollow cylinders, about $25 \mathrm{~nm}$ in diameter and up to several micrometers long. Found in eukaryotic cells, i.e., those that contain genetic material within a nucleus, microtubules contribute to cell motion and shape maintenance (Purves et al., 2001).

The length of microtubules increases and decreases dynamically by addition and removal of molecules of tubulin protein. Tubulin molecules are added at the ends of microtubules in a process called polymerization. Microtubules are used to impart a rigid structure to cells, e.g., at cell extensions, and depolymerization, or removal of tubulin molecules, collapses this rigid structure (Purves et al., 2001).

\section{i. Extracting strategy from biological system}

Many microtubules originate from a microtubuleorganizing center (MTOC), which in certain types of cells, is markedly at the center of the cell. It is suspected that the MTOC centers itself by extending microtubules that "scout out the cell periphery" (Lodish et al., 2000), as shown in Figure 4.
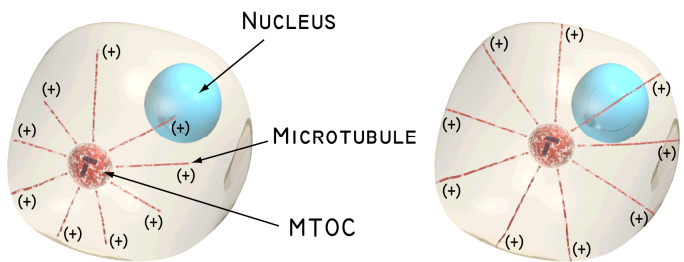

\section{Figure 4. Centering of MTOC by extension of microtubules towards cell boundary. Modified from Lodish et al. 2000 by T. Nissen (Shu et al., 2003).}

\section{ii. Applying strategy to engineering system}

The centering strategy used by the MTOC can be implemented directly, e.g., by extending samelength spines from an object to be centered within a gripping volume.

A colleague at the Technical University of Denmark proceeded to develop solutions for the microassembly centering problem using the MTOC centering strategy. These concepts are shown in Figure 5. Figure 5A shows the function of the temporary spines being fulfilled using $\mathrm{CO}_{2}$ ice, which is to be sublimated following positioning of the part. Figure 5B shows a looser interpretation of the MTOC strategy, i.e., extending the spines from the gripper rather than the object. The flat gripper with flaps can be fabricated using a combination of an elastic material and a shape memory alloy. The shape memory alloy can be configured to reach a prescribed position after reaching a particular temperature. The elastic material can then be used to return the flaps to the flat position by decreasing the temperature (Shu et al., 2003).

Bütefisch and Büttgenbach (2001) report that mechanical grippers typically use two opposing arms or fingers. The advantage of using more than two fingers is the improved ability to accurately position non-symmetric objects.

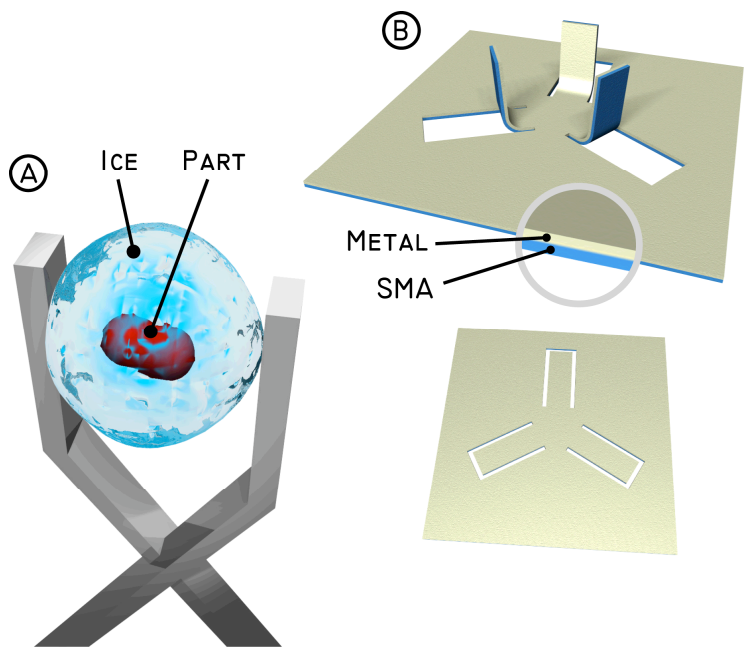

Figure 5. Possible implementations of MTOC-centering strategy by $T$. Lenau. Figure by T. Nissen (Shu et al., 2003).

\section{Natural language processing}

Despite the advantages, there are challenges involved with using natural-language knowledge sources to identify biological analogies. A primary difficulty involves the quantity and quality of matches.

Although a single text is currently used as the source, there can be an unmanageable number of matches to the keywords, including synonyms, troponyms, etc. Some of the matches are irrelevant and have the following characteristics, described by Hacco and Shu (2002) and Chiu and Shu (2004).

\section{a. Part of speech and word sense}

One situation that leads to irrelevant matches is when the intended part of speech of the keyword searched does not match the part of speech of the occurrence of the keyword in the text. This is especially problematic when the primary sense, or meaning, of a word differs greatly between its noun and verb forms. 
For example, to find biological analogies for sealing a joint, a logical keyword with which to start is 'seal'. However many occurrences of 'seal' in Purves et al. (2001) involve the marine mammal seal. A solution to this problem that can be easily implemented is to tag each word of the corpus with its part of speech, e.g., by using the Brill tagger (Brill, 1995). In this way, all occurrences of 'seal' in the noun form can be disregarded. However, this would also remove any occurrences of 'seal' in the noun form that has the desired sense related to joining and joints, e.g., "Endodermal cell walls contain suberin, which forms a waterproof seal wherever it is present." In this example, although the found occurrence of 'seal' is a noun, it is still relevant to the verb form sought. The problem here is one of "word sense disambiguation", i.e., distinguishing which sense is intended in a particular use of a word, and is still an active area of research in computer science.

\section{b. Abstract objects}

For problems that are concerned with physical objects, e.g., repairing and handling physical parts, matches where the grammatical object of a keyword verb is an abstract entity tend not to be useful. For the remanufacture example, a match describing a process to 'correct an equation' is less useful than the match for the self-correcting action of fainting.

WordNet, a lexical database in which words are organized according to relationships with other words, as opposed to alphabetically (Miller, 1993), is useful to us in multiple ways. First, WordNet lists both synonyms and troponyms that can be used to generate alternative keywords to find matches. WordNet can also be used to determine if a word, e.g., the grammatical object of a keyword verb is an abstract or physical entity.

Due to the potentially large number of matches to keywords, current work underway seeks to determine dominant biological phenomena associated with keywords by word frequency analysis (Chiu and Shu, 2004).

\section{Human factors in using analogies}

Another challenge in using biological analogies for design involves the human process of extracting relevant strategies from the biological phenomena and applying these strategies to design problems.

Observations of people performing these processes revealed four types of similarities between the biological phenomena presented and the concepts developed using them as stimuli (Mak and Shu, 2004a). These four similarity types, literal implementation, biological transfer, analogy and anomaly, will be illustrated using an example problem from Dieter (2000):

Develop ways to eliminate puddles from pedestrian walkways after a rainstorm.

Solutions for this problem will be generated using the following description of a biological phenomenon from Purves et al. (2001) as stimulus:

\begin{abstract}
A parasitic leech makes an incision in its host to expose its blood. It can ingest so much blood in a single feeding that its body may enlarge several times. A substance secreted by the leech into the wound keeps the host's blood flowing. Leeches are used to reduce fluid pressure and prevent blood clotting in damaged tissues and to eliminate pools of coagulated blood.
\end{abstract}

\section{a. Literal implementation}

An example of a literal implementation would be, "Use leeches to ingest puddles on walkways." In this type of similarity relationship, the strategy used is the one presented in the description, but the same biological forms, leeches, are used to perform the necessary actions to carry out the strategy.

\section{b. Biological transfer}

An example of a biological transfer can be expressed as, "Use leeches to fill up depressions in sidewalks so that puddles don't form." In this case, the strategy presented in the description is not used, but the biological forms, leeches, are transferred into a different strategy in the proposed solution.

\section{c. Analogy}

Analogies represent the type of similarity intended in biomimetic design by using the strategy in the phenomenon without transferring the biological forms. Analogous concepts propose suction- and/or absorption-based solutions that implement the strategy used by leeches, without using actual leeches in the implementation.

\section{d. Anomaly}

Anomalies do not actually involve any obvious similarity between the concept and the description used as stimulus. An example of an anomalous concept could be expressed as, "develop a material that reacts with air to clot puddles on sidewalks." Some anomalous concepts result from 
misunderstanding of the biological phenomenon. Others result from fixating on certain words in the description that is not representative of the overall strategy, e.g., 'clotting.'

Current work seeks to reduce this and other types of fixation that were observed in people attempting to develop analogous concepts from descriptions of biological phenomena (Mak and Shu, 2004b).

\section{Summary}

This paper summarized our past and current work towards developing a methodology to identify and use relevant biological analogies for any given engineering problem in a systematic manner. Analogies identified using the chosen approach for case studies in design for remanufacture and microassembly were described. Finally discussed were ongoing challenges and work in language analysis and human factors in using biological phenomena to develop analogous solutions.

\section{Acknowledgments}

Gratefully acknowledged is the financial support of Natural Sciences and Engineering Research Council of Canada (NSERC).

\section{References}

Brill, E., 1995, Transformation-Based Error-Driven Learning and Natural Language Processing: A Case Study in Part-of-Speech Tagging, Computational Linguistics, 21/4: 543-565.

Bütefisch, S., Büttgenbach, S., 2001, New DifferentialType SMA Actuator for a Miniature Silicon Gripper, Proc. of SPIE, Vol. 4235:102-107.

Chiu, I., Shu, L., 2004, Natural Language Analysis for Biomimetic Design, Proc. of 2004 ASME Design Engineering Technical Conferences and Computers and Information in Engineering Conference, Salt Lake City, Utah, Sept. 28-Oct. 2, 2004, DETC2004/DTM-57250.

Dieter, G.E., 2000, Engineering Design: A Materials and Processing Approach - 3rd edition, McGraw-Hill.

Friedberg, E.C., Walker, G.C., Siede, W., 1995, DNA Repair and Mutagenesis, ASM Press, Washington, D.C.

Hacco, E., Shu, L., 2002, Biomimetic Concept Generation Applied to Design for Remanufacture, Proc. of ASME Design Engineering Technical Conferences and
Computers and Information in Engineering Conference, Montreal, Sept. 29-Oct. 2, 2002, DETC2002/DFM-34177.

Lodish, H., Berk, A., Zipursky, S.L., Matsudaira, P., Baltimore, D., Darnell, J. 2000, Molecular and Cell Biology, 4/e, W.H. Freeman \& Co., New York.

Mak, T.W., Shu, L.H., 2004a, Abstraction of Biological Analogies for Design, CIRP Annals, Vol. 53/1, In press.

Mak, T., Shu, L., 2004b, Use of Biological Phenomena in Design by Analogy, Proc. of 2004 ASME Design Engineering Technical Conferences and Computers and Information in Engineering Conference, Salt Lake City, Utah, Sept. 28-Oct. 2, 2004, DETC2004/DTM-57303.

Miller, G.A., 1993, Introduction to WordNet: An Online Lexical Database, Five Papers on WordNet, pp. 1-25.

$\mathrm{ftp} / / / \mathrm{ftp} . \operatorname{cogsci}$.princeton.edu/pub/wordnet/5papers.ps

Purves W.K., Sadava, D., Orians, G.H., Heller, H.C., 2001, Life, The Science of Biology, 6/e, Sinauer Associates, Sunderland, MA.

Shu, L. and Flowers, W., 1999, Application of a Design-for-Remanufacture Framework to the Selection of Product Life-Cycle Fastening and Joining Methods, International Journal of Robotics and Computer Integrated Manufacturing (Special Issue: Remanufacturing), 15/3:179-190.

Shu, L.H., Lenau, T.A., Hansen, H.N., Alting, L., 2003, Biomimetics Applied to Centering in Microassembly, CIRP Annals, 52/1:101-104.

Shu, L.H., 2004, Biomimetic Design for Remanufacture in the Context of Design for Assembly, Proceedings of the I Mech E Part B: Journal of Engineering Manufacture, 218/3:349-352. (Invited Paper)

Vakili, V., Shu, L, 2001, Towards Biomimetic Concept Generation, Proc. of 2001 ASME Design Technical Conferences, Design Theory and Methodology Conference, Pittsburgh, PA, Sept. 9-12, 2001, DETC2001/DTM-21715.

Vincent, J., Mann, D., 2002, Systematic Technology Transfer from Biology to Engineering, Philosophical Trans. of The Royal Society: Physical Sciences, 360: 159-173.

Williams, J., 2001, Quantification and Analysis of Remanufacturing Waste Streams for Improving Product Design, Master of Applied Science Thesis, University of Toronto, Department of Mechanical and Industrial Engineering. 